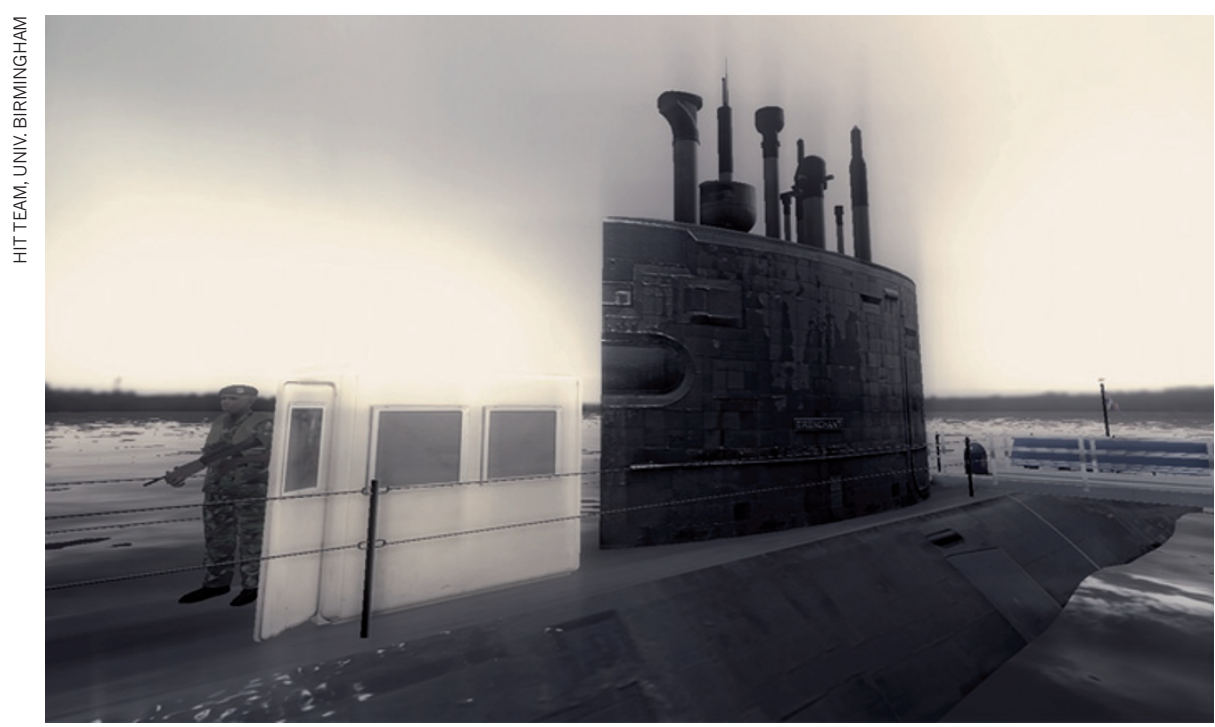

The SubSafe software creates a virtual submarine to train personnel to find safety equipment.

Q\&A Robert Stone

\title{
The virtual trainer
}

Robert Stone, director of the Human Interface Technologies Team at the University of Birmingham, UK, develops 'serious games' for training soldiers and sailors. He explains how immersion in virtual worlds can prepare military personnel for their experiences on the battlefield - and help them to heal after they return.

\section{How useful are virtual environments in military training?}

Even with today's technology, we can't fully replace the experience of live combat or military activities. But where current training practices fall short, virtual environments or serious gaming can fill the gap.

\section{What sort of environments do you model?}

We train a range of military recruits - from new submariners to those who will patrol areas to locate improvised explosive devices

in Afghanistan and the United Kingdom. One project involves a virtual British town. We program it for different scenarios - say, a suspect package left at a railway station. Army instructors work with trainees to pick and place objects, such as the command vehicle, cordons or robot disposal vehicles; they then discuss appropriate questions to ask the police or witnesses, to build up a strong picture of the developing incident.

\section{Is virtual training proven to work?}

Yes. For example, SubSafe is a three-dimensional recreation of a nuclear submarine,

$\rightarrow$ NATURE.COM

For a Nature video of

Robert Stone's work:

go.nature.com/puuxso built using gametechnology software. Naval instructors use it to train submariners, particularly in locating safety equipment - fire extinguishers, breathing apparatus and thermal imagers. When we evaluated the software with trainees at the naval base in Devonport, UK, over 18 months, we found that there was a significant improvement in the real-world performance of the guys who got the software, compared with those who did not. That's important because in the future, the United Kingdom will have fewer submarines, which means less time for trainees on real vessels.

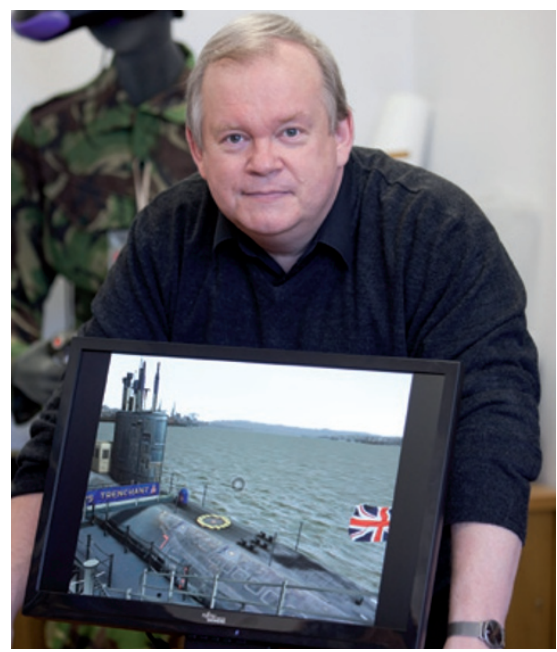

Robert Stone and his training program SubSafe.

\begin{tabular}{|l|l|}
\hline BEYOND THE BOMB & $\begin{array}{l}\text { BEN } \\
\text { Science and the military } \\
\text { nature.com/military }\end{array}$ \\
\hline
\end{tabular}

Can your work help soldiers who return from combat?

We plan to use the technology to provide restorative environments for returnees who have had serious surgery, such as amputations. Display screens at the ends of their hospital beds would show a view of sea- and landscapes in real time: evidence suggests that exposure to blue-green environments - such as seascapes and meadows - improves recovery and reduces the need for analgesics. Virtual environments will be used as part of therapeutic 'guided imagery' and relaxation techniques. We also aim to help amputees to prepare their remaining muscles for prosthetic devices, using motion-sensing devices in tandem with virtual environments - so that they can virtually walk along a beach, scuba-dive, skim stones or throw a frisbee.

\section{What are the challenges for an academic working with the military?}

It is a difficult area to break into. These projects are not something you can perform in splendid academic isolation; you have to go into the field and understand the jobs that these guys do. I've flown on helicopters, been to sea in submarines and fired small Gatling guns off the side of Royal Navy ships. There are risks - I once experienced a helicopter gearbox failure, and have been marooned on the bottom of a Scottish loch in a damaged three-person submersible.

Does it help that most recruits are familiar with computer games?

Yes; the games culture is helping us to manage the expectations and engagement of future military-simulation users. Many young recruits have been put in front of simulators using older technologies, and have said: "We can do better than this at home." That way, you lose hearts and minds instantly. But if you show them something that looks the part, such as the Call of Duty series of action games, you instantly have their attention.

\section{Are some people still sceptical?}

That never goes away. It's not just the older members of the armed forces; we get it from younger officers as well. Fortunately, when they have seen the results, most of them come around very quickly. It is all part of the challenge. 